\title{
Double-Layered Matrix of Shellac Wax-Lutrol in Controlled Dual Drug Release
}

\author{
Thawatchai Phaechamud ${ }^{1,2}$ and Chai-ek Choncheewa ${ }^{1}$
}

\begin{abstract}
Received 26 September 2015; accepted 9 December 2015; published online 22 December 2015
Abstract. Double-layered matrix tablets prepared from shellac wax-lutrol were fabricated using a molding technique, and the release of hydrochlorothiazide and propranolol $\mathrm{HCl}$ from the inner tablet or outer layer was studied. The simultaneous determination of dual drug release was measured with first derivative UV spectrophotometry. The tablet containing shellac wax as the outer tablet and lutrol as the inner tablet showed more appropriate drug release and the size of the inner layer influenced the rate of drug release. In addition, the aqueous solubility of the drug and the components of the inner tablet or outer layer affected the drug release behavior. Most of the double-layered tablets exhibited the drug-release pattern which fitted well with zero-order kinetic due to the restriction of the release surface. Biphasic drug release pattern was found in the tablet of which the outer layer rapidly eroded. The drug dissolution data from drug-loaded-outer layer could predict the dissolution time for the outer layer of drug-loaded inner part of double-layered matrix tablet. Incorporation of lutrol increased the drug release from shellac wax matrix, and the zero-order release was attained by fabricating it into a double-layered tablet.
\end{abstract}

KEY WORDS: double-layered matrix; dual drug release; lutrol; shellac wax; tablet.

\section{INTRODUCTION}

Various methods are used to prepare a matrix tablet such as wet granulation, dry granulation, direct compression, and also a sintering technique. The sintering technique is defined as the bonding of adjacent particle surfaces in a mass of powder or in a compact by the application of heat. This technique is interesting because it uses less process and excipient (1). Many heating techniques have been used to prepare the matrix tablet such as hot-melt extrusion when the drug and other excipients are mixed and blended under high temperature and extruded whereas injection molding also uses injection at a high pressure and temperature to prepare the matrix tablet $(2,3)$. The major drawback of these techniques is the requirement for high cost machinery. An easier way to prepare the matrix tablet is by a melting and molding technique as recently reported which the matrix tablet was fabricated from polyethylene glycol (PEG) using various mold sizes for modulating the release of indomethacin $(4,5)$.

Typically, the lipid matrices promote a sustainable drug release. In some case, however, the drug release is too slow to maintain the drug concentration at an appropriate level or reach the therapeutic concentration. Therefore, a hydrophilic polymer is incorporated to mix or melt together with these hydrophobic matrices to tune up the drug release. Poloxamer or lutrol (L) is copolymers containing ethylene oxide (EO) and propylene oxide (PO) blocks arranged in a triblock

\footnotetext{
${ }^{1}$ Department of Pharmaceutical Technology, Faculty of Pharmacy, Silpakorn University, Nakorn Pathom, 73000, Thailand.

${ }^{2}$ To whom correspondence should be addressed. (e-mail: thawatchaienator@gmail.com)
}

structure presenting amphiphilic properties which promoted the release of theophylline from a glyceryl palmitostearate capsule $(6,7)$. The hydrophilic property of $\mathrm{L}$ created the pores and channels in the wax matrix allowing higher dissolution medium penetration into the matrix. Drug release from surface eroding devices with various geometries has been investigated (8-13). Shellac wax $(\mathrm{S})$ is used in agricultural manufacture for fruit or vegetable coating $(14,15)$. In the pharmaceutical field, shellac is applied as a compression coating for conventional tablets (16). S and L are moldable materials; therefore, they can be fabricated into a tablet using a molding technique by melting and cooling. The hydrophilic polymer can tune up the drug release profile of a waxy matrix because it can create pores and channels on the wax matrix to allow the penetration of dissolution medium $(6,17)$. The addition of $\mathrm{L}$ to modulate the drug release of monolithic $\mathrm{S}$ tablet has been reported previously (7) which the sustainable drug release was obtained. Therefore, the understanding of the drug release from double-layered matrix tablets prepared from $\mathrm{S}$ and $\mathrm{L}$ was performed in this study using propranolol $\mathrm{HCl}$ (PRO) and hydrochlorothiazide (HCT) as hydrophilic and hydrophobic model drugs, respectively. Both drugs are used together to treat hypertension as the combined formulation in a marketed product named Inderide ${ }^{\circledR}$.

The wax matrix tablet is an interesting approach to control the drug release; however, the drug release might be too low because of its hydrophobicity. The hydrophilic polymer is therefore incorporated into the wax matrix to tune up the drug release. However, the drug release might not exhibit a zeroorder kinetic which is the desired drug release kinetic of this pharmaceutical dosage form. Geometric shapes and surface area play an important role relating to that release. Double- 
layered matrix tablets were therefore prepared with $\mathrm{S}$ and $\mathrm{L}$ loaded with PRO and HCT and restrictions of surface area on dual drug release patterns were studied. These tablets were prepared by the alteration of the inner and outer layers of each matrix base which has not previously been reported. The effect of drug loading at the outer or inner layers was also investigated.

\section{MATERIALS AND METHODS}

\section{Materials}

Hydrochlorothiazide (HCT) (batch No I 1413891, supplied by Government of Pharmaceutical Organization, Thailand), propranolol HCl (PRO) (lot no M080311, PC Drug Co., Ltd., Bangkok, Thailand), lutrol F127 (L) (lot no WPDF563B, BASF, Ludwigshafen, Germany), and shellac wax (Ake shellac Co., Ltd., Lumpang, Thailand) were used as received.

\section{Preparation of Matrix Tablet}

$\mathrm{L}$ and $\mathrm{S}$ were accurately weighed after deducting the displacement value (D.V.) of each drug ( $80 \mathrm{mg}$ of PRO and HCT/tablet). The D.V. of each drug was calculated by using the equation as described previously (18). The schematic configuration for the obtained double layer tablet is shown in Fig. 1 and the total formula and their compositions are shown in Table I. The inner drug-loaded matrix (IDM) was fabricated by firstly melting and molding the selected component in 8or $12-\mathrm{mm}$ diameter stainless steel molds with the melting method at $80^{\circ} \mathrm{C}$. HCT and PRO were combined and incorporated into the molten base. $\mathrm{L}$ and 7:3 (7:3 L:S) were used to prepare the inner tablet. $\mathrm{S}$ was not incorporated with these drugs due to its hydrophobic property which did not allow the drug to be released. However, it was still used as control base because of its good barrier property. The inner tablet was carefully placed at the center of the $15-\mathrm{mm}$ diameter stainless steel mold and thereafter the outer layer material (S, L or 7:3) was individually melted at $80^{\circ} \mathrm{C}$ and poured around the inner tablet. Double-layered tablets were solidified at room

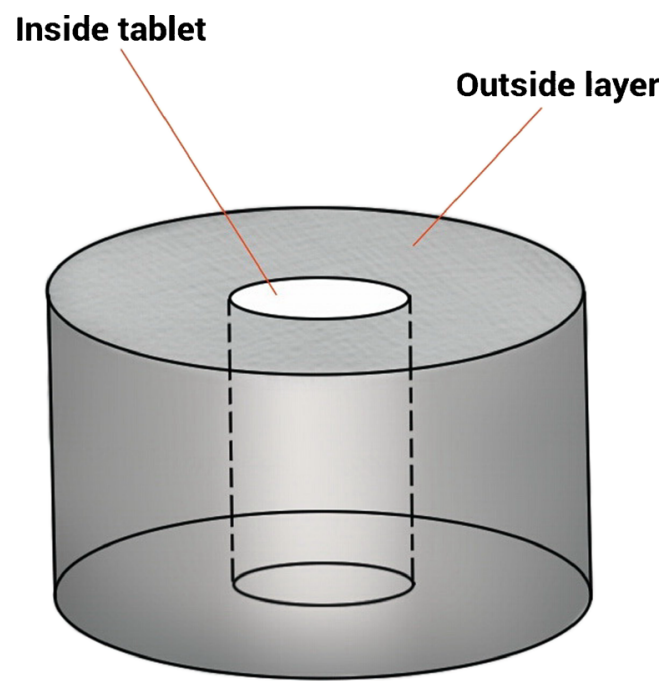

Fig. 1. View of double-layered tablet comprising inner tablet (8 or $12 \mathrm{~mm}$ ) with outer layer $(15 \mathrm{~mm})$
Table I. Formulation and Composition of Each Layer for DoubleLayered Tablets

\begin{tabular}{lll}
\hline $\begin{array}{l}\text { Mold diameter } \\
\text { (inner/outer) }\end{array}$ & Drug loading & $\begin{array}{l}\text { Matrix base } \\
\text { (inner/outer) }\end{array}$ \\
\hline $8 \mathrm{~mm} / 15 \mathrm{~mm}$ and $12 \mathrm{~mm} / 15 \mathrm{~mm}$ & Inner & $\mathrm{L} / \mathrm{L}$ \\
& (IDM) & $\mathrm{L} / \mathrm{S}$ \\
& & $\mathrm{L} / 7: 3$ \\
& & $7: 3 / \mathrm{L}$ \\
& & $7: 3 / \mathrm{S}$ \\
& & $7: 3 / 7: 3$ \\
& Outer & $\mathrm{L} / \mathrm{L}$ \\
& (ODM) & $\mathrm{S} / \mathrm{L}$ \\
& & $7: 3 / \mathrm{L}$ \\
& & $\mathrm{L} / 7: 3$ \\
& & $\mathrm{~S} / 7: 3$ \\
& & $7: 3 / 7: 3$ \\
\hline
\end{tabular}

temperature and kept in a desiccator. Additionally, the drugloaded outer matrix (ODM) was also prepared using only an 8 -mm tablet as the inner tablet. The inner tablet was carefully placed in the center of the $15-\mathrm{mm}$ diameter stainless steel mold before the molten base incorporating both model drugs was poured around the inner tablet. The obtained ODM were solidified at room temperature and kept in the desiccator before testing.

Weight Variation, Friability, Hardness, Thickness, and Diameter Determination

Weight variations of the tablets were determined which average weight and standard deviation were calculated $(n=20)$. The friability was determined as the percent weight loss from 20 tablets. Twenty tablets were weighed and rotated for 100 revolutions for $4 \mathrm{~min}$ using a friabilator (Yieheng Engineering, Bangkok, Thailand). Ten tablets were observed for their hardness, thickness, and diameter using a hardness tester (TBH 325 TD, Basel, Switzerland). Average and standard deviation of hardness, thickness, and diameter were presented $(n=10)$.

\section{Drug Release Study}

The dissolution of PRO or HCT was studied using dissolution apparatus I (basket apparatus, RC-6, Minhua Pharmaceutical machinery Co., LTD., China) at $100 \mathrm{rpm}$ rotational speed in $900 \mathrm{~mL}$ distilled water at $37.0 \pm 0.5^{\circ} \mathrm{C}$ as previously reported (7). The 5-mL dissolution medium was sampled at specific time intervals. The volume of sample solution removed was replaced with an equal volume of fresh dissolution fluid. The simultaneous determination of dual dissolution for the two drugs was measured using first derivative UV spectrophotometry (FUV) (Perkin-Elmer, Germany) as previously reported $(7,19)$ and the obtained spectra (D1), at 297 and $336 \mathrm{~nm}$ for PRO and HCT, respectively, were employed for this study. The range of linearity of PRO and HCT was 1.5-7.5 $\left(r^{2}=0.9999\right)$ and $3.6-18.0 \mu \mathrm{g} / \mathrm{ml}\left(r^{2}=0.9996\right)$, respectively. The $\%$ recovery of PRO and HCT was 106.59 and 97.11, respectively. Precision was determined as intraday and interday 
precision. The RSD of intraday precision was 2.46 and $1.88 \%$ for PRO and HCT, respectively. For interday precision, the RSD was 2.23 and $1.57 \%$ for PRO and HCT, respectively. The LOD of the standard curve was found to be 0.10 and $0.49 \mu \mathrm{g} /$ $\mathrm{ml}$ for PRO and HCT, respectively. The LOQ was 0.31 and $1.48 \mu \mathrm{g} / \mathrm{ml}$ for PRO and HCT, respectively. The dual drug release was also conducted in $\mathrm{HCl}$ buffer $\mathrm{pH} 1.2$ (HBS) and phosphate buffer $\mathrm{pH} 6.8$ (PBS). The cumulative percentage drug release of each system was fit with first order, zero-order, Higuchi's, cube root, and Korsmeyer-Peppas equations using Scientist for Windows (version 2.1). Least square fitting method was used for dissolution profile fitting to different release mathematical models by the nonlinear computer program, Scientist for Windows, version 2.1, MicroMath Scientific Software, Salt Lake City, UT, USA) (20). Goodness-of-fit was evaluated using the coefficient of determination $\left(r^{2}\right)$ the Model Selection Criterion (msc) (MicroMath Scientist Handbook Rev, 1995). The higher the value of $r^{2}$ and msc indicated the greater goodness-of-fit.

\section{Statistical Analysis}

All statistical significance was examined by using one-way analysis of variance (ANOVA) followed by the least significant difference (LSD) post hoc test with significance level of $p<0.05$. The analysis was performed by using SPSS for windows (version 11.5).

\section{RESULTS}

\section{Physical Properties of Double-Layered Matrix Tablets}

The prepared tablet fabricated with this molding technique at $80^{\circ} \mathrm{C}$ did not change the physicochemical properties of drug molecules in matrices as previously reported (21). The previous article from my previous work indicated that there was no change for drug molecules. The double-layered tablet was prepared from three different matrix bases including L, S, and 7:3. The combined drug was loaded in $L$ or $7: 3$ because $S$ did not allow drug release. However, $\mathrm{S}$ showed a good barrier property against the dissolution medium, hence it was chosen to prepare a non-drug-loaded layer and compared with $\mathrm{L}$ and 7:3. Both drugs were loaded into the outer (ODM) or inner (IDM) sections of the double-layered tablets. For fabricating IDM, the molds with diameters of 8 and $12 \mathrm{~mm}$ were used. In the case of ODM, only the $8-\mathrm{mm}$ inner tablet was used. The physical properties of 8 and $12 \mathrm{~mm}$ IDM are presented in Table II. The obtained data were compared between the tablets prepared with different bases using either the inner or outer of the matrix tablets and the dimension of the inner layer which was either 8 or $12 \mathrm{~mm}$. The inner layers were made from $\mathrm{L}$ or 7:3. These inner tablets were covered by an outer layer which was made of $\mathrm{L}, \mathrm{S}$, or 7:3. The diameter of tablet was in rank of $14.78-15.03 \mathrm{~mm}$ and thickness was in rank of $6.56-6.85 \mathrm{~mm}$. The difference in tablet weight was influenced by the type of matrix base used either inner tablet or outer layer. For tablets having the same material for the inner matrix, the type of material used in the outer layer influenced the tablet weight. The tablet weight was highest in tablets with outer layer made of $\mathrm{L}$, followed by 7:3 and $\mathrm{S}$, respectively. This trend was similar for both 8 - and 12-mm
IDMs. The tablet weight differed slightly and depended on whether L or 7:3 was used as the inner matrix base. The inner tablet made of $\mathrm{L}$ was heavier than that made of 7:3. This result was more evident when the inner tablet was larger. All of prepared mold tablets showed the apparently low friability $(0.04-0.18 \%)$. For thickness and diameter, the differences were minor due to the fix diameter of mold.

The hardness of IDMs made from both 8 and 12-mm inner tablets seemed highest when 7:3 was the outer layer followed by $\mathrm{S}$ and $\mathrm{L}$, respectively. The inner tablet also affected the hardness but it was more evident in 12-mm IDMs. By comparison for inner tablets made from $\mathrm{L}$ and 7:3, the hardness was not quite different in the $8-\mathrm{mm}$ IDMs. The inner tablet made from 7:3 showed higher hardness than that made from L. The hardness of 12-mm IDMs was higher than that of 8-mm IDMs as shown in Table II. However, the effect of L and $\mathrm{S}$ as outer layers on tablet hardness was not found when $7: 3$ was used as the inner tablet as reported by the statistical results. For 12-mm L/7:3 and L/S IDMs, the hardness was not different but that of the other formula was different significantly $(p<0.05)$.

In the case of ODMs, their physical properties are shown in Table II. The weight of tablets made from $\mathrm{L}$ as the outer layer was higher than those made from 7:3. The effect of the inner material on tablet weight demonstrated the same trend as observed in the outer layers. Tablet weights were highest when the inner layer was $L$ followed by 7:3 and S, respectively. The hardness depended on the type of both outer and inner layer bases. The hardness of tablets made from 7:3 as outer layer was higher than that made from L. The tablet hardness of the inner tablet made from $S$ and 7:3 was not quite different unlike $\mathrm{L}$ inner tablet which showed the lowest hardness. The hardness for ODMs was not different between the tablets made from $7: 3$ and $S$ as the inner tablet as seen in the statistical analysis data for the pair of $\mathrm{S} / \mathrm{L}-7: 3 / \mathrm{L}$ and $\mathrm{S} / 7: 3-7: 3 / 7: 3$ tablets.

\section{Drug Release from Double-layered Matrix Tablet}

\section{$I D M$}

Distilled water was used as dissolution medium to avoid the effect of $\mathrm{pH}$ and ion from medium on drug release. For 8mm IDMs, both HCT and PRO were released within $180 \mathrm{~min}$ from $\mathrm{L} / \mathrm{L}$ whereas $\mathrm{L} / \mathrm{S}$ sustained drug release by delaying the dissolution of both drugs while the drug release was steady at approximately $420 \mathrm{~min}$ (Fig. 2). Biphasic drug release was found in $L / 7: 3$. The first phase was between 0 and $150 \mathrm{~min}$ when both drugs released at a slower rate than the second phase. The maximum HCT release of $\mathrm{L} / \mathrm{L}$ was higher than from $\mathrm{L} / \mathrm{S}$ and $\mathrm{L} / 7: 3$, respectively. PRO released faster than HCT from all formula. The fastest drug release was obtained from $\mathrm{L} / \mathrm{L}$ followed by $\mathrm{L} / 7: 3$ and slowest from $\mathrm{L} / \mathrm{S}$. A sustained drug release was found for all formula when the inner tablet was 7:3 (Fig. 2). Biphasic drug release was obtained from 7:3/L and $7: 3 / 7: 3$. However, the initial phase of drug release from $7: 3 / \mathrm{L}$ was shorter than $7: 3 / 7: 3$ when the second phase started at about $240 \mathrm{~min}$. The drug release from 7:3/S was not biphasic but with a unique release pattern when the initial drug release was faster, then there was a slow drug release. The drug release from $\mathrm{L}$ inner tablet was faster than that of 7:3. The 
Table II. Physical Properties of 8- and 12-mm IDMs and 8-mm ODM

\begin{tabular}{|c|c|c|c|c|c|c|}
\hline & 8-mm IDM & & 12-mm IDM & & 8-mm ODM & \\
\hline Inner/outer & Weight $(\mathrm{mg})^{*}$ & Hardness** (Newton; N) & Weight $(\mathrm{mg})^{*}$ & Hardness** (Newton; N) & Weight $(\mathrm{mg})^{*}$ & $\begin{array}{l}\text { Hardness** } \\
\text { (Newton; N) }\end{array}$ \\
\hline $\mathrm{L} / \mathrm{L}$ & $1291.3 \pm 8.8$ & $100.50 \pm 9.80$ & $1296.2 \pm 17.1$ & $140.10 \pm 11.55$ & $1297.6 \pm 7.1$ & $108.70 \pm 8.84$ \\
\hline $\mathrm{L} / \mathrm{S}$ & $1077.6 \pm 13.0$ & $134.10 \pm 15.42$ & $1141.4 \pm 38.5$ & $165.70 \pm 23.51$ & - & - \\
\hline $\mathrm{L} / 7: 3$ & $1213.1 \pm 9.0$ & $160.20 \pm 20.69$ & $1220.9 \pm 21.6$ & $171.20 \pm 20.60$ & $1207.8 \pm 16.3$ & $139.90 \pm 13.45$ \\
\hline 7:3/L & $1286.6 \pm 10.9$ & $112.10 \pm 9.77$ & $1274.7 \pm 8.1$ & $177.50 \pm 13.71$ & $1270.0 \pm 13.2$ & $158.70 \pm 9.15$ \\
\hline $7: 3 / \mathrm{S}$ & $1061.8 \pm 16.2$ & $109.40 \pm 20.69$ & $1120.7 \pm 14.2$ & $200.30 \pm 34.52$ & - & - \\
\hline $7: 3 / 7: 3$ & $1198.6 \pm 14.8$ & $176.10 \pm 14.56$ & $1244.1 \pm 12.9$ & $250.40 \pm 25.88$ & $1172.5 \pm 9.5$ & $170.70 \pm 7.04$ \\
\hline $\mathrm{S} / \mathrm{L}$ & - & - & - & - & $1259.3 \pm 11.7$ & $157.40 \pm 15.15$ \\
\hline $\mathrm{S} / 7: 3$ & - & - & - & - & $1155.3 \pm 7.1$ & $172.80 \pm 16.48$ \\
\hline
\end{tabular}

The determined values are mean $\pm \mathrm{SD}(* n=20 ; * * n=10)$

outer layer also influenced the drug release with the fastest drug release being found when the outer layer was $L$ followed by $7: 3$ and $S$, respectively.

For IDMs with a 12-mm inner layer using $\mathrm{L}$ as the inner layer, the drug release from tablets prepared with $\mathrm{L} / \mathrm{L}$ and L/7:3 were almost the same (Fig. 2). The drugs were completely released within approximately $150 \mathrm{~min}$. Biphasic drug release was found only in the case of HCT. The second phase of drug release was evident after 45 and $120 \mathrm{~min}$ for $\mathrm{L} / \mathrm{L}$ and $\mathrm{L} / 7: 3$, respectively. In the case of $\mathrm{L} / \mathrm{S}$, a sustained release pattern was reached at about $300 \mathrm{~min}$. The drug release was fastest for both $\mathrm{L} / \mathrm{L}$ and $\mathrm{L} / 7: 3$ and slowest for L/S. For IDMs with $12 \mathrm{~mm}$ inner layers prepared with $7: 3$, the fastest release was found for 7:3/L followed by 7:3/7:3 and slowest in 7:3/ $\mathrm{S}$, respectively (Fig. 2). Biphasic drug release was found with the transition period at about 60 and $90 \mathrm{~min}$ for $7: 3$ / $\mathrm{L}$ and 7:3/7:3, respectively. According to the results, the inner and outer layers influenced the drug release. The inner layer made from 7:3 sustained the drug release more effectively than that made from L. The drug releases were not quite different for IDMs using the inner tablet as 7:3 and $\mathrm{S}$ or $7: 3$ as the outer layer. These formulations sustained the drug release longer than the tablet using $\mathrm{L}$ as outer layer. On the contrary, the drug release from the $\mathrm{L} / \mathrm{L}$ and $\mathrm{L} / 7: 3$ released at the same rate and faster than $\mathrm{L} / \mathrm{S}$ when $\mathrm{L}$ was the inner tablet.

This study compared the drug release from systems comprising of 8 and 12-mm inner layers. Normally, an inner tablet with smaller surface area should sustain the drug release more effective than one with a larger surface area (12). Therefore, the 8-mm IDM should sustain the drug release longer than the 12-mm IDM. Our results indicate that, in addition to surface area of the inner compartment, the type of matrix also influenced the drug release rate. When $\mathrm{L}$ was employed as the inner tablet, the 8-mm IDM could sustain the drug release more effectively than the 12-mm IDM except for L/L when the release rates of 8- and 12-mm IDM seemed equal. On the other hand, when 7:3 was used as the inner tablet, the $12-\mathrm{mm}$
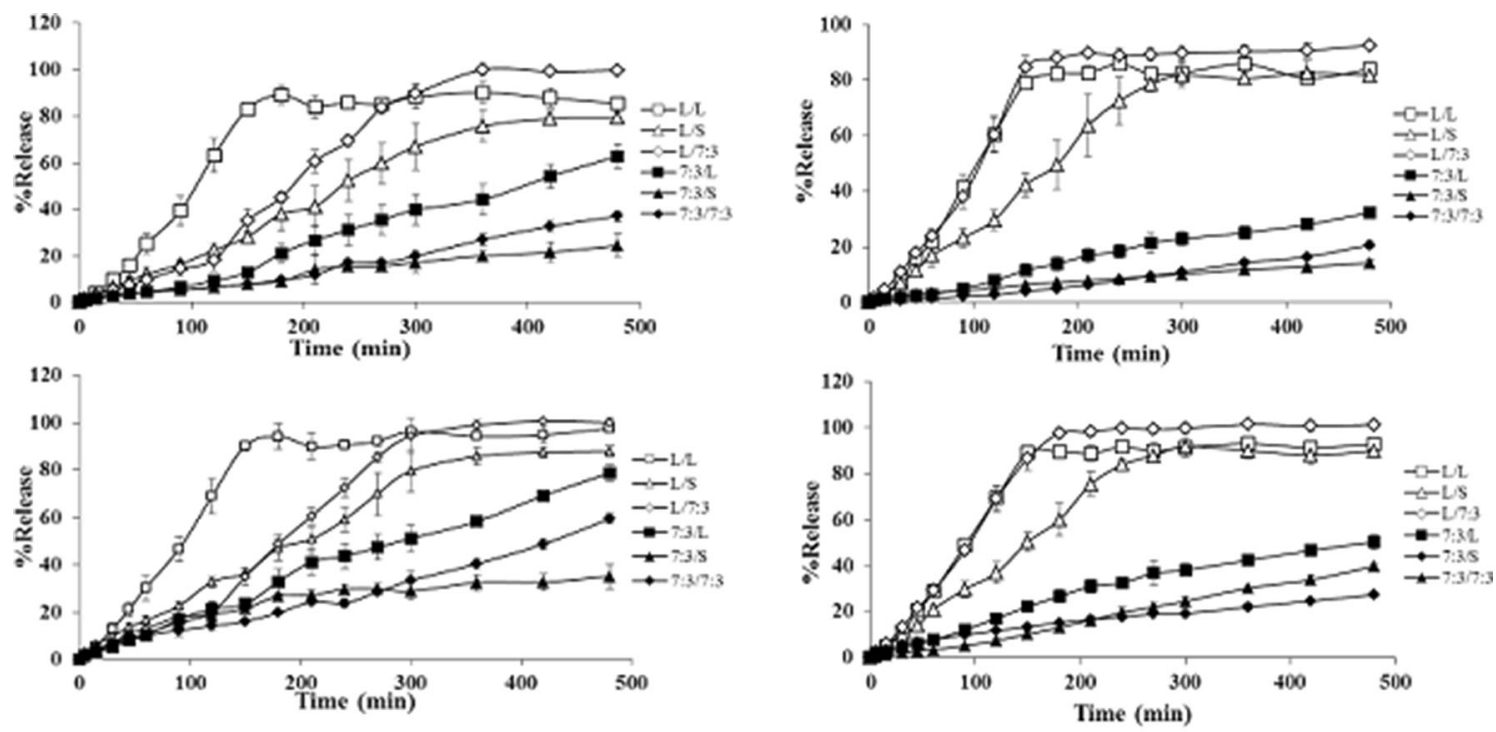

$8 \mathrm{~mm}$ IDM

$12 \mathrm{~mm}$ IDM

Fig. 2. Drug release profiles of HCT (above) and PRO (below) from 8 and 12-mm IDM formula with different inner tablets and outer layers in distilled water $(n=3)$. The $\neg-,-\triangle$, and $\neg-$ expressed the outer layer as L, S, and 7:3 when L was the inner tablet, respectively. The $\neg-\multimap$, and $\multimap$ expressed the outer layer as L, S, and 7:3 when 7:3 was the inner tablet, respectively 
IDM showed a slower drug release than the 8-mm IDM, due to the swelling of the 7:3 L:S tablet as seen during the dissolution test.

\section{$O D M$}

Eight millimeter L, S, and 7:3 tablets without drugs were used as the core layer, and the drugs were incorporated into the outer layer prepared from 7:3 and L. The type of inner tablet did not influence the drug release when the outer layer was $\mathrm{L}$ while $\mathrm{L}$ or $\mathrm{S}$ was used as the inner tablet (Fig. 3). The drugs released at the same rate except for $7: 3 / \mathrm{L}$ when the drug release was apparently more sustainable than that from both $\mathrm{L} / \mathrm{L}$ and $\mathrm{S} / \mathrm{L}$. Almost all of drug in $\mathrm{L} / \mathrm{L}$ and $\mathrm{S} / \mathrm{L}$ released within $90 \mathrm{~min}$ but $7: 3 / \mathrm{L}$ promoted a slower drug release. The core layer did not influence the drug release when 7:3 was employed as the outer layer. The type of base used as the outer layer influenced the drug release (Fig. 3). The outer layer made from 7:3 sustained the drug release longer than that made from L. On the other hand, the tablet containing $\mathrm{L}$ as the outer layer released most of its drug content within $90 \mathrm{~min}$ for $\mathrm{L} / \mathrm{L}$ and $\mathrm{S} / \mathrm{L}$ but longer for 7:3/L. When compared with IDM, the drug released from ODM was apparently faster when the same inner tablet was used. Moreover, biphasic drug release was not observed in ODM systems.

\section{Effect of Dissolution Medium on Drug Release}

The prepared tablet which sustained the drug release at the most constant rate was selected to study the effect of $\mathrm{pH}$ on the drug release. Using this criterion, the 8-mm IDM of L/S
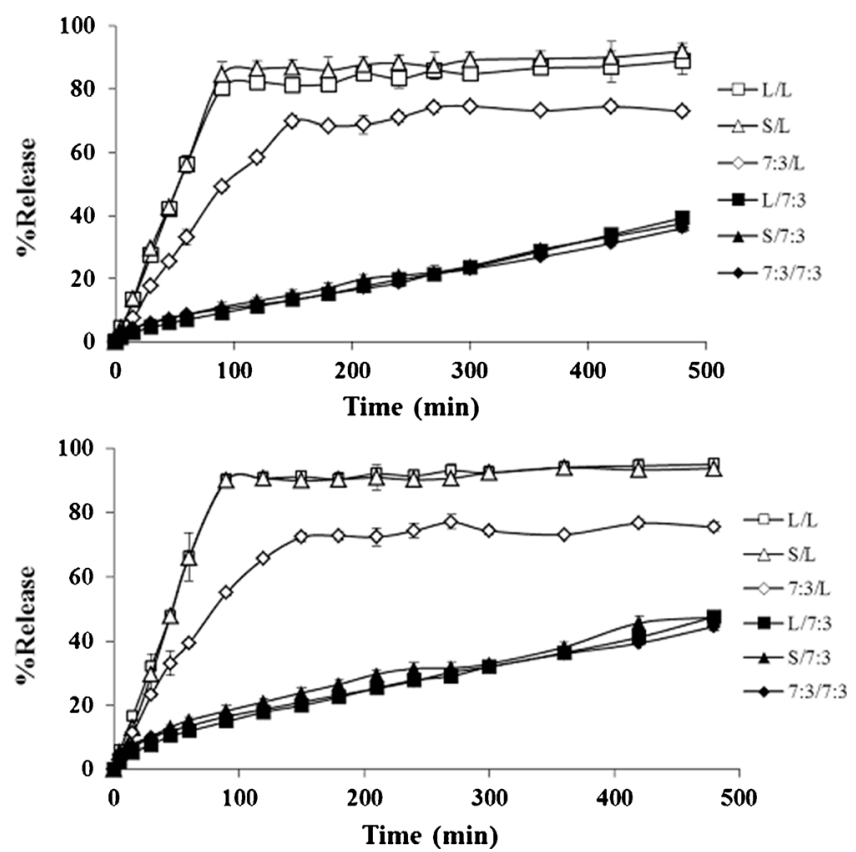

Fig. 3. Drug release profiles of HCT (above) and PRO (below) from ODM formula with different inner tablets and outer layers in distilled water $(n=3)$. The $\neg \square,-\Delta$, and $\neg-$ expressed the outer layer as L, S, and 7:3 when $\mathrm{L}$ was the inner tablet, respectively. The - - , $\multimap$, and $\multimap$ expressed the outer layer as L, S, and 7:3 L:S when 7:3 was the inner tablet, respectively was chosen because the drug release was steady and the drug release was complete in about $420 \mathrm{~min}$. The release of both PRO and HCT could be sustained for up to 420 min (Fig. 4). However, the highest drug released in HBS was lower than that released in distilled water. The rate and content of PRO released was higher than that of HCT in distilled water but the rate and content of $\mathrm{HCT}$ released was higher than PRO in HBS.

The release of both drugs was sustained for $480 \mathrm{~min}$ in PBS. The different drug releases were found in HBS and distilled water because HCT released in HBS faster than in distilled water and PBS but PRO released in HBS slower than that found in distilled water and slightly faster than PBS after $180 \mathrm{~min}$. The release rate of HCT in PBS was close to that in distilled water in the first $150 \mathrm{~min}$. The release rate of PRO in PBS was similar to that in HBS in the initial state. Then, HCT released in distilled water gradually faster than that found in PBS. HCT released faster than PRO in PBS and vice versa in distilled water.

\section{Drug Release Patterns from Double-Layered Matrix Tablet}

General mathematical equations were developed to describe the drug release from slabs, spheres, and infinite cylinders (8). The double-layered matrix tablet was designed to take advantage of the surface area controlling the drug release since it was designed to keep the surface area constant throughout the dissolution study. The IDM had smaller surface area than ODM; thus, it sustained the drug release longer. Because of the constant surface area, it should release at a zero-order kinetic $(11,12)$; however, the system prepared with some formula did not. Thus, other variables beside surface area must be taken into consideration.
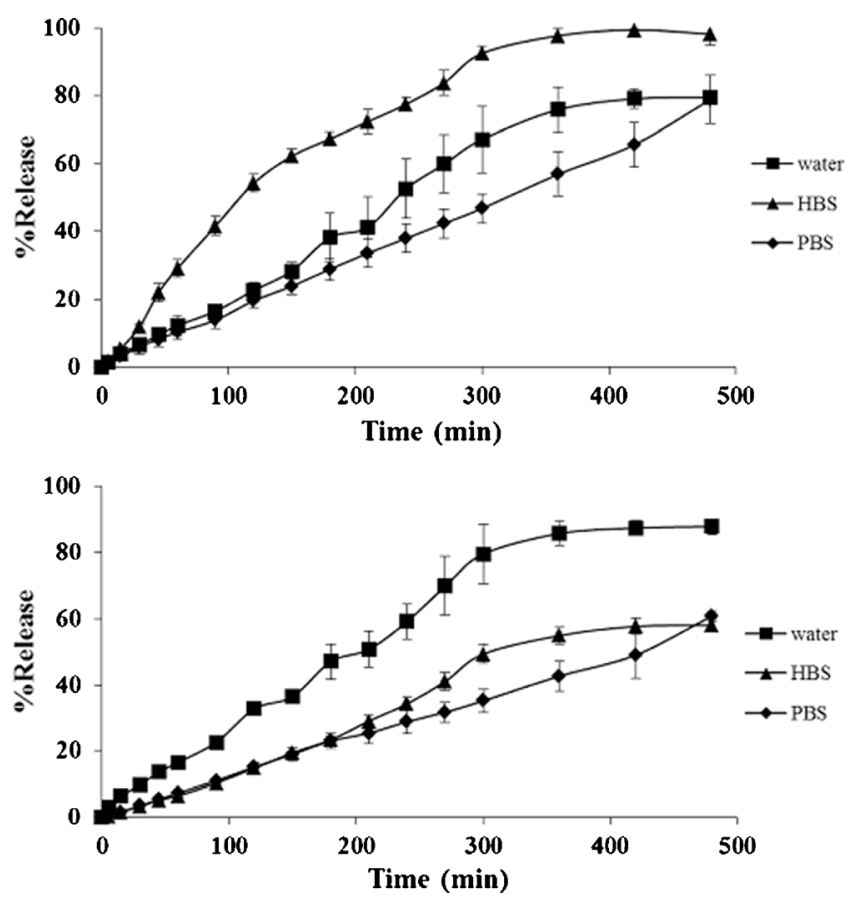

Fig. 4. Drug release profiles of HCT (above) and PRO (below) from 8 -mm IDM of $\mathrm{L} / \mathrm{S}$ in different dissolution fluids $(n=3)$ 
$I D M$

The degrees of goodness-of-fit of release profiles for both HCT and PRO from 8-mm IDMs to different mathematical equations are shown in Table III. Most of the drug release profiles fitted well with zero order except for that of HCT from 7:3/L which followed a first-order kinetic and PRO from 7:3/S which was suitably described by Higuchi's model.

The drug release profile from $12 \mathrm{~mm}$ IDMs was influenced by the increased surface area. Both PRO and HCT releases still fitted well with the zero-order kinetic but that from the 7:3 inner tablet formula varied (data not shown). The HCT of 7:3/S and 7:3/L releases followed Higuchi's model $\left(r^{2}=0.9959\right.$ and 0.9920 , respectively). The PRO release profile from 7:3/L was suitably described with Higuchi's model $\left(r^{2}=0.9967\right)$ but 7:3/S and 7:3/7:3 fitted well with the first order $\left(r^{2}=0.9944\right.$ and 0.9981 , respectively).

\section{$O D M$}

Almost all of the drug release from ODM fitted well with the zero-order kinetic especially for HCT loaded tablets (Table IV). The cube root law only fitted well for HCT released from 7:3/L. The first order suitably described the drug release from both $\mathrm{L} / 7: 3$ and $7: 3 / \mathrm{L}$ but the cube root law best described that from 7:3/7:3. The influence of an increased surface area on drug release was more apparent in PRO than in HCT. Drugs loaded in 7:3 L:S exhibited various release patterns. Most of the drug loaded in $\mathrm{L}$ showed the best fit with the zero-order kinetic.

\section{Drug Release Patterns in Different pH}

The release pattern from 8-mm IDMs of L:S showed best fit with the zero order for both PRO and HCT in distilled water but the release profile of some drug in PBS or HBS did not follow the zero-order kinetic (data not shown). The release pattern of HCT in HBS pH 1.2 was first order; however, it remained zero kinetic in PBS $\mathrm{pH}$ 7.4. For PRO, the $\mathrm{pH}$ did not influence the dissolution profile. The zero order still remained the best fitted model for both drugs released in HBS and PBS.

\section{DISCUSSION}

The hardness of double-layered tablets varied depending on many factors such as the ratio of $\mathrm{L}$ to $\mathrm{S}$, the size of the inner tablet, and the thickness of the outer layer. The chemical structure arrangement and type of polymer, whether thermoset or thermoplastic, influenced the hardness of the injection molded tablet (22). The larger inner tablet indicating a thinner outer layer produced a higher hardness; therefore, the inner tablet played the more important role relating to tablet hardness. Actually, a thicker outer layer should produce higher hardness as previously reported (23). They investigated the physical properties of compressed three-layered tablets where the increasing amount of barrier layer produced higher tablet hardness. This might be due to the differences in tablet preparation where the hardness of the molded tablet depended on the molecular arrangement of the compounds in the inner tablet.

The double-layered tablet in this study was designed to modulate the drug release with a zero-order profile and to prolong the drug release. The design of the tablet focused mainly on the controlling surface area of the matrix tablet. Many tablets have been designed to control the surface area for drug release such as the donut or ring shaped (12) or other shapes such as core-in-cup and compressed coated tablets as previously reported (24). The design of tablets in this experiment was based on the restriction of surface area. The drugloaded matrix was used as the inner tablet then the surface around the inner tablet was shielded by the outer layer without drug loading. Therefore, the inner drug-loaded matrices (IDMs) allowed drug release mainly from the top and bottom of the tablets only. The outer drug-loaded matrices (ODMs) were also investigated in order to study the sustained drug

Table III. Comparison of Degrees of Goodness-of-Fit from Curve Fitting of Dissolution Profiles of HCT and PRO from 8-mm IDM with Different Inner Tablets and Outer Layers (Inner/Outer) in Distilled Water

\begin{tabular}{|c|c|c|c|c|c|c|c|c|c|c|c|}
\hline \multirow[t]{2}{*}{ Formula } & \multicolumn{2}{|c|}{ Zero order } & \multicolumn{2}{|c|}{ First order } & \multicolumn{2}{|c|}{ Higuchi's } & \multicolumn{2}{|c|}{ Cube root } & \multicolumn{3}{|c|}{ Korsmeyer-Peppas } \\
\hline & $r^{2}$ & $\mathrm{msc}$ & $r^{2}$ & $\mathrm{msc}$ & $r^{2}$ & $\mathrm{msc}$ & $r^{2}$ & $\mathrm{msc}$ & $r^{2}$ & $\mathrm{msc}$ & $n$ \\
\hline \multicolumn{12}{|l|}{ HCT } \\
\hline $\mathrm{L} / \mathrm{L}$ & 0.9906 & 4.00 & 0.9351 & 2.07 & 0.8735 & 1.67 & 0.9573 & 2.49 & 0.9976 & 5.04 & 1.47 \\
\hline $\mathrm{L} / \mathrm{S}$ & 0.9905 & 4.26 & 0.9606 & 2.83 & 0.9574 & 2.76 & 0.9764 & 3.35 & 0.9951 & 4.72 & 1.00 \\
\hline $\mathrm{L} / 7: 3$ & 0.9760 & 3.23 & 0.9351 & 2.16 & 0.8926 & 1.56 & 0.9614 & 2.59 & 0.9895 & 3.70 & 1.24 \\
\hline $7: 3 / \mathrm{L}$ & 0.9846 & 3.78 & 0.9905 & 4.26 & 0.9636 & 2.91 & 0.9884 & 4.01 & 0.9913 & 4.07 & 0.67 \\
\hline $7: 3 / \mathrm{S}$ & 0.9868 & 3.76 & 0.9865 & 3.73 & 0.9544 & 2.59 & 0.9746 & 3.10 & 0.9869 & 3.47 & 1.06 \\
\hline $7: 3 / 7: 3$ & 0.9899 & 4.09 & 0.9859 & 3.64 & 0.9045 & 1.95 & 0.9889 & 4.00 & 0.9899 & 3.84 & 1.01 \\
\hline \multicolumn{12}{|l|}{ PRO } \\
\hline $\mathrm{L} / \mathrm{L}$ & 0.9962 & 4.78 & 0.9393 & 2.14 & 0.9585 & 2.18 & 0.9633 & 2.64 & 0.9996 & 6.80 & 1.29 \\
\hline $\mathrm{L} / \mathrm{S}$ & 0.9906 & 4.26 & 0.9540 & 2.72 & 0.9234 & 2.12 & 0.9696 & 3.13 & 0.9952 & 4.80 & 1.33 \\
\hline $\mathrm{L} / 7: 3$ & 0.9560 & 2.62 & 0.8822 & 1.64 & 0.8889 & 1.53 & 0.9465 & 2.26 & 0.9121 & 1.68 & 1.99 \\
\hline $7: 3 / \mathrm{L}$ & 0.9891 & 4.19 & 0.9790 & 3.53 & 0.8926 & 1.90 & 0.9880 & 4.09 & 0.9925 & 4.40 & 0.81 \\
\hline $7: 3 / \mathrm{S}$ & 0.8290 & 1.43 & 0.8577 & 1.62 & 0.9110 & 2.09 & 0.8482 & 1.55 & 0.9652 & 2.86 & 0.30 \\
\hline $7: 3 / 7: 3$ & 0.9804 & 3.60 & 0.9458 & 2.58 & 0.9520 & 2.54 & 0.9588 & 2.86 & 0.9975 & 5.49 & 2.00 \\
\hline
\end{tabular}


Table IV. Comparison of Degrees of Goodness-of-Fit from Curve Fitting of Dissolution Profiles of HCT and PRO from ODM Formula with Different Inner Tablets and Outer Layers (Inner/Outer) in Distilled Water

\begin{tabular}{|c|c|c|c|c|c|c|c|c|c|c|c|}
\hline \multirow[t]{2}{*}{ Formula } & \multicolumn{2}{|c|}{ Zero order } & \multicolumn{2}{|c|}{ First order } & \multicolumn{2}{|c|}{ Higuchi's } & \multicolumn{2}{|c|}{ Cube root } & \multicolumn{3}{|c|}{ Korsmeyer-Peppas } \\
\hline & $r^{2}$ & $\mathrm{msc}$ & $r^{2}$ & $\mathrm{msc}$ & $r^{2}$ & $\mathrm{msc}$ & $r^{2}$ & $\mathrm{msc}$ & $r^{2}$ & $\mathrm{msc}$ & $n$ \\
\hline \multicolumn{12}{|l|}{ HCT } \\
\hline $\mathrm{L} / \mathrm{L}$ & 0.9992 & 6.27 & 0.9613 & 2.45 & 0.9047 & 1.55 & 0.9809 & 3.18 & 0.9994 & 6.19 & 0.94 \\
\hline $\mathrm{S} / \mathrm{L}$ & 0.9977 & 5.29 & 0.9757 & 2.92 & 0.9057 & 1.56 & 0.9908 & 3.89 & 0.9995 & 6.39 & 0.86 \\
\hline $7: 3 / \mathrm{L}$ & 0.9897 & 3.91 & 0.9959 & 4.82 & 0.9860 & 3.60 & 0.9983 & 5.81 & 0.9969 & 4.79 & 0.68 \\
\hline $\mathrm{L} / 7: 3$ & 0.9955 & 5.04 & 0.9905 & 4.29 & 0.9579 & 2.80 & 0.9927 & 4.55 & 0.9961 & 4.94 & 1.17 \\
\hline $\mathrm{S} / 7: 3$ & 0.9958 & 5.07 & 0.9861 & 3.91 & 0.9428 & 2.46 & 0.9903 & 4.24 & 0.9994 & 6.80 & 1.54 \\
\hline 7:3/7:3 & 0.9960 & 5.16 & 0.9885 & 4.11 & 0.9437 & 2.47 & 0.9915 & 4.41 & 0.9990 & 6.33 & 1.44 \\
\hline \multicolumn{12}{|l|}{ PRO } \\
\hline $\mathrm{L} / \mathrm{L}$ & 0.9903 & 3.84 & 0.9689 & 2.58 & 0.9548 & 2.30 & 0.9869 & 3.54 & 0.9960 & 4.31 & 0.79 \\
\hline $\mathrm{S} / \mathrm{L}$ & 0.9938 & 4.28 & 0.9608 & 2.44 & 0.9578 & 2.36 & 0.9832 & 3.29 & 0.9968 & 4.55 & 0.82 \\
\hline $7: 3 / \mathrm{L}$ & 0.9691 & 2.90 & 0.9984 & 5.85 & 0.9844 & 3.54 & 0.9946 & 4.66 & 0.9966 & 4.83 & 0.58 \\
\hline $\mathrm{L} / 7: 3$ & 0.9820 & 3.73 & 0.9877 & 4.11 & 0.9811 & 3.68 & 0.9868 & 4.05 & 0.9894 & 4.12 & 0.67 \\
\hline $\mathrm{S} / 7: 3$ & 0.9975 & 5.68 & 0.9939 & 4.79 & 0.9645 & 3.03 & 0.9961 & 5.23 & 0.9977 & 5.62 & 0.93 \\
\hline $7: 3 / 7: 3$ & 0.9968 & 5.42 & 0.9981 & 5.96 & 0.9813 & 3.67 & 0.9985 & 6.22 & 0.9988 & 6.22 & 0.80 \\
\hline
\end{tabular}

release from outer shell when the inner was restricted. Moreover, drug release profiles obtained from the ODMs could describe the disappearance of the outer layer of IDM which promoted the biphasic release profiles.

The 8-mm IDM showed a faster drug release for the $\mathrm{L}$ inner tablet whereas the IDM using a 7:3 inner tablet showed a slower drug release. This was due to the rapid erosion of the hydrophilic matrix of $\mathrm{L}(25,26)$. In the case of the $7: 3$ outer layer, the release was sustained but still faster than the $S$ outer layer because 7:3 lowered the drug release owing to the apparently strengthened gel as previously reported (7). The gel structure of L occurred by the rearrangement of PPO and PEO unimer of polymer chain. In the dissolution medium, the PPO firstly dehydrated and formed the inner layer micelle then PEO formed outer layer micelle due to its hydrophilic property. The spherical micelle was then attributed packing each other if it contained sufficient polymer to become a gel $(26,27)$. The gel network from high content of $\mathrm{L}$ was owing to hexagonal form formation which was the compact structure. However, it still did not completely block the surface area around the inner tablet; hence, it showed greater drug release than S. Biphasic drug release was found in $\mathrm{L} / 7: 3,7: 3 / \mathrm{L}$, and 7:3/7:3 tablets. Since the outer shell could completely erode and, subsequently, the surface area around the inner tablet was suddenly increased, the result was an increase in the later phase of drug release as illustrated in Fig. 5a. Biphasic drug release was evident for the tablet which its release surface area was changed (24). When the outer layer was insoluble, S, the surface area around inner tablet, did not alter. Therefore, L/S and 7:3/S did not exhibit biphasic drug release. S completely protected the surface area around the inner tablet; thus, the drug release mainly occurred from the top and bottom of IDM as presented in Fig. 5b. L/L rapidly eroded because the erosion rate of the same type of polymer was not different; therefore, the biphasic drug release was not evident. Therefore, the type of outer shell influenced the drug release from IDMs. The similar result had been reported previously for the influence of coated layers onto Geomatrix or coated matrices on drug release behavior which the biphasic release profile was evident $(13,28,29)$.

Drug release from 12-mm IDMs using L or 7:3 as the inner tablet was faster than that of 8-mm IDM tablets because of the greater surface area on the top and bottom of $12-\mathrm{mm}$ IDMs. The drug release of $\mathrm{L}$ inner tablets varied depending on the outer layer. The release profile was similar when the outer layer was L or 7:3 but S produced a greater prolongation of drug release than the other two types of outer layer. This result was different from that of the $8-\mathrm{mm}$ IDM where the

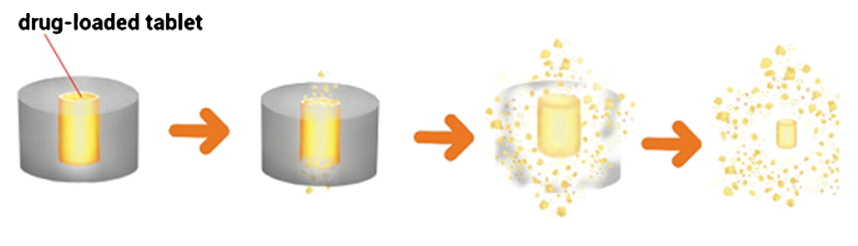

a
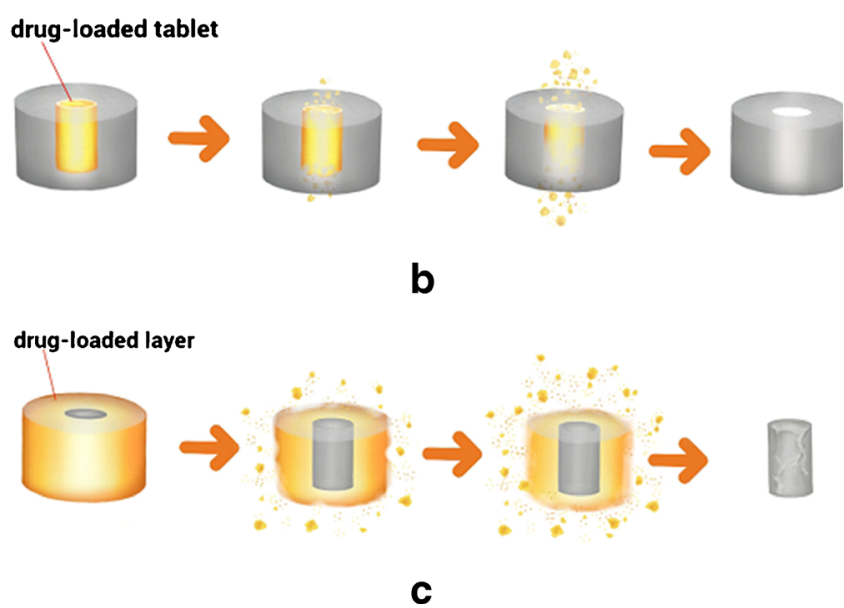

Fig. 5. Schematic depiction of drug release from a IDM with erodible inner tablet and outer layer; b IDM with erodible inner tablet, and c ODM with erodible outer layer 
release was fastest when the outer layer was L, followed by 7:3 and $S$, respectively. Therefore, the outer layer made from $7: 3$ did not apparently influence the sustained drug release in 12mm IDMs because the thin outer layer caused rapid erosion. Biphasic drug release was also found in 7:3/L and 7:3/7:3 IDMs where the shift point occurred at 60 and $90 \mathrm{~min}$, respectively; therefore, L outer layer almost eroded within $60 \mathrm{~min}$ and the 7:3 within $90 \mathrm{~min}$. The outer shell did not erode when $\mathrm{S}$ was the outer shell; thus, the surface area was lesser than those of the previous two formulations. The type and hydrophilicity of barrier influenced the drug release from Geomatrix tablet (29). Triple-layered donut-shaped tablets for extending theophylline release were prepared by layering with compression of bottom and top layers with water insoluble polymer such as ethyl cellulose (13).

For ODMs, drug release was almost complete at only $90 \mathrm{~min}$ for a drug-loaded L formula. Sustained drug release was found for tablets using $\mathrm{L}$ as the outer layer in 7:3/L ODM which prolonged the drug release for up to $150 \mathrm{~min}$. Small amounts of the drug might have contaminated the 7:3 inner layer from the preparation process; hence, the drug release was slower. The drug released at almost the same rate for $\mathrm{L} / 7: 3, \mathrm{~S} / 7: 3$, and 7:3/7:3 because the surface area played a more significant role on drug release (30). The surface area of ODMs was greater than that of IDMs and the swellable 7:3 could obstruct the hole being necessary for the erosion of the inner tablet especially when $\mathrm{L}$ was used as the inner tablet. The hole obstructed by the swelling of the outer shell was reported in the donut-shaped tablet (13). For $S / 7: 3$ and 7:3/7:3, the inner tablet did not erode when $\mathrm{S}$ was the inner tablet and gradually eroded when 7:3 was used as the inner tablet. The slow erosion of the 7:3 inner tablet was overcome by the swelling of the outer layer, as in L/7:3. According to the results, the surface area for drug release of ODMs using $7: 3$ as the outer shell was not significantly changed thus the drug release profiles from these tablets was not quite different. The drug release patterns of ODMs comprising erodible outer layers and insoluble inner tablets are presented in Fig. 5c.

HCT released into HBS faster than into distilled water and PBS. On the other hand, the release of PRO into distilled water was fastest whereas a slower drug release was evident in the two remaining buffers. PRO dissolution was lowered by the effect of salts in the dissolution medium, especially common ion effect like chloride ion in HBS (31). A major role in the decrease of PRO dissolution was from the common ion effect and ionic strength influencing the dissolution of ionized drugs $(32,33)$. The variation in HCT release in this experiment was due to the difference of gel structure in the matrix tablet during dissolution. In HBS, the gel structure was loosened by hydrochloride as previously described (34); therefore, HCT in HBS released faster than in PBS and distilled water. PRO released in distilled water faster than in the other two buffers because the dissolution of the drug was decreased by the common ion in the latter two dissolution fluids. Although the loosened gel network occurred in HBS, the drug dissolution might play a more important role on drug release, thus the release of PRO in HBS and PBS was slower than in distilled water.

Typically, a constant surface area promoted a zero-order kinetic (11-13). However, the hydrophilicity of a drug also influenced the release behavior as seen for HCT from 7:3/L and PRO from 7:3/S 8-mm IDM when the release data fitted well with the first-order and Higuchi's model, respectively. The outer shell of 7:3/L was rapidly eroded; therefore, the surface area was not kept constant throughout the experiment hence the HCT release depended on its concentration. On the other hand, PRO from 7:3/L still released with a zero-order kinetic although the surface area was not kept constant. The faster release of PRO might balance with the release surface area since the drug was loaded into a smaller tablet compared with the single-layer tablet; hence, the surface area was small. The smaller release area compensated for the high water soluble property of PRO and promoted the zero-order release of PRO loaded in a 7:3/L IDM. In the case of 7:3/S, PRO release fitted well with the Higuchi's model. As discussed previously, the zero-order release kinetic of PRO loaded in 7:3/L was owing to the balance between the release surface area and the hydrophilic property of PRO. When the surface area around the tablet was shielded with $S$, the surface area of drug release was only at the top and bottom of the tablet. Unfortunately, the swelling of the top and bottom of IDM as mentioned previously (7) generated the dumb bell-like tablet. Because of the increasing dissolution path length, PRO release fitted well with Higuchi's model. In the case of 7:3/S, the release of HCT was slower than that of PRO. Therefore, the drug release was mainly controlled by the erosion of the tablet and fitted well with the zero-order kinetic. The difference of drug release characteristic of multilayered tablets depended on hydrophilicity of lower and upper barriers (35). In general, a constant drug release should be achieved when both barrier layers are hydrophilic and the core is hydrophobic (36-38). However, other factors also need to be considered to attain a zero-order drug release.

Most of the drug release from the $8-\mathrm{mm}$ IDM was also fitted well with a zero-order kinetic but the larger tablet (12$\mathrm{mm}$ IDM) showed more varied drug release kinetics. This variety of drug release occurred only when the drug was loaded in 7:3 because the surface area was not kept constant due to the swelling of 7:3 tablet. The different drug release kinetic from the same base was also explained by the hydrophilicity of each drug. PRO dissolved and diffused easier than $\mathrm{HCT}$; thus, the variety of drug release mechanisms was obtained. The drug release kinetic of PRO in 7:3/L was Higuchi's because the surface area was not kept constant and rapidly eroded. The first-order release kinetic was obtained when the outer layer was $S$ or 7:3. The 7:3 inner tablet promoted swelling of the matrix tablet (7); therefore, the release area on the top and bottom was not constant; hence, the drug release did not fit with a zero-order kinetic. As described previously, the high water solubility of PRO promoted the variation of drug release. In the cases of 7:3/S and 7:3/7:3, the swelling of the inner tablet and the high water solubility of PRO resulted in the first-order release kinetic. Unlike HCT, its release kinetic was Higuchi's model for 7:3/L and 7:3/S because it slowly released. Therefore, the increase of diffusion path length played a significant role on its release behavior $(39,40)$.

The high release area of ODM was presented because the drug was loaded in the outer layer. Therefore, the kinetic of drug release was varied especially when the drug was loaded in 7:3. Moreover, the hydrophilicity of PRO promoted many drug release kinetics for different formulations unlike HCT where the drug release fitted well with the zero-order kinetic. 
PRO release from L/7:3 and 7:3/L followed first order because of the presence of a higher surface area and the high water solubility of PRO. The system could not keep the drug concentration gradient constant because the high surface area promoted higher penetration of the dissolution medium. Therefore, PRO released from these two formulations was described suitably by the first order. Some matrix tablets liberated the drug with anomalous non-Fickian diffusion kinetic has been reported previously $(41,42)$. In the case of drug release from 7:3/7:3, the release of PRO followed the cube root law indicating drug release from erosion with a constant geometric shape whereas the HCT release best fitted with the zero-order kinetic because its slow release was balanced with matrix erosion. HCT was less water soluble than PRO; moreover, it could be solid dispersed in L. Therefore, it was possible that the release of HCT was mainly controlled by the erosion of the matrix tablet and followed the zero-order kinetic. Otherwise, the drug liberated from the loosened gel structure caused the alteration of the drug release kinetic which was discussed later in the effect of $\mathrm{pH}$ variation. For the reasons described above, HCT released from this system was best described by the zero-order kinetic. In the case of PRO, the drug released faster because of its high water soluble property; hence, the drug release did not fit well with the zero-order kinetic. However, the release was restricted by the compact and dense structure of the gel. Therefore, the suitable model for explanation of drug release of PRO was the cube root law.

The drug release profiles of 8-mm IDMs comprising L/S almost fitted well with the zero-order kinetic except for that of HCT in HBS medium. As described previously, HCT release was mainly controlled by the erosion of the matrix base because it was solid dispersed into L; hence, it promoted the zero-order release kinetic. In case of HCT in HBS, the gel network was loosened by this medium as mentioned above (34). Therefore, the HCT easily liberated from the matrix base and the drug release was not mainly controlled by the erosion of the matrix but from the drug dissolution. According to the result, the release of HCT in HBS depended on its concentration described by the first-order kinetic. The loosening of the gel network should have produced easier diffusion of PRO and resulted in the first-order kinetic release profile the same as HCT. The release of naproxen through some hydrogel followed the anomalous transport (43). However, the drug release kinetic of PRO in this experiment still fitted well with zero order. PRO dissolution was decreased by the other salts in the buffer medium; therefore, instead of rapidly dissolving and diffusing out of the tablet, PRO gradually dissolved and diffused with a zero-order drug release kinetic.

\section{CONCLUSIONS}

The restriction of surface area of double-layered tablets as IDMs could promote a zero-order drug release. Most of the drug release from these tablets fitted well with zero order whereas some tablets did not because of the swelling of the 7:3 inner tablet. Biphasic drug release was evident when the outer layer eroded rapidly resulting in the appearance of the surface around the drug-loaded inner tablet and promoting the later higher drug release rate. A comparison of the drug release from different sizes of the inner tablet demonstrates that increasing the release surface area promoted a faster drug release. L/S IDMs sustained the dual drug release with a zeroorder kinetic owing to the restriction of surface area of the drug-loaded inner matrix by the outer insoluble $\mathrm{S}$. When compared with IDMs, the drug release from ODMs was faster when the same inner tablet was used. Moreover, there was no biphasic drug release from ODM systems. The drug dissolution data from ODMs could indicate the dissolution time of the outer layer of IDM. Solubility of drugs influenced their release behavior from the double-layered tablets. The increased HCT release in HBS was explained by the depletion of the gel network in this medium. PRO released fastest in distilled water whereas the common ion effect and the other salts in the buffer medium promoted a low dissolution of PRO. S, which is a natural product obtained as waste from the shellac manufacturing process, could be used as a matrix base. The drug release from $S$ matrix tablets could be tuned up by the incorporation of a hydrophilic polymer such as L. Zero-order drug release could be achieved by the restriction of drug release surface area as in the form of double-layered tablet.

\section{ACKNOWLEDGMENTS}

This research work was kindly supported by the Higher Education Research Promotion and National Research University (NRU \& HERP), Office of the Higher Education Commission, Thailand, grant No. SURDI (57/01/02 (HERP)). The authors are thankful to Dr. Purin Charoensuksai for his assistance and support. We are also grateful for technical support from the Research and Development Institute, Silpakorn University. This research work was also facilitated by the Faculty of Pharmacy, Silpakorn University, Thailand.

\section{REFERENCES}

1. Tiwari BM, Khare S, Mishra V, Bhargava S. Matrix tablet: a potential drug carrier for oral drug delivery. J Pharm Res. 2012;5:2448-56.

2. Chokshi R, Hossein Z. Hot-melt extrusion technique: a review. Iran J Pharm Res. 2004;3:3-16.

3. Quinten T, Beer DT, Vervaet C, Remon JP. Evaluation of injection moulding as a pharmaceutical technology to produce matrix tablets. Eur J Pharm Biopharm. 2009;71:145-54.

4. Mesnukul A, Yodkhum K, Phaechamud T. Solid dispersion matrix tablet comprising indomethacin-PEG-HPMC fabricated with fusion and mold technique. Indian J Pharm Sci. 2009;71:413-20.

5. Mesnukul A, Yodkhum K, Mahadlek J, Phaechamud T. Characterization of indomethacin release from polyethylene glycol tablet fabricated with mold technique. Indian J Pharm Sci. 2010;72:92-100.

6. Jannin V, Pochard E, Chambin O. Influence of poloxamers on the dissolution performance and stability of controlled-release formulations containing precirol ${ }^{\circledR}$ ATO 5. Int J Pharm. 2006;309:6-15.

7. Phaechamud T, Choncheewa C. Sole and dual drug release patterns from shellac wax-jutrol matrix tablets fabricated with fusion and molding technique. Indian J Pharm Sci. 2015;77:62-74.

8. Karasulu HY, Ertan G. Different geometric shape hydrogel theophylline tablets: statistical approach for estimating drug release. Il Farmaco. 2002;57:939-45.

9. Siepmann J, Kranz H, Peppas NA, Bodmeier R. Calculation of the required size and shape of hydroxypropyl methylcellulose matrices to achieve desired drug release profiles. Int J Pharm. 2000;201:151-64. 
10. Gohel MC, Panchal MK. A novel mathematical method for quantitative expression of deviation from zero-order drug release. Pharm Technol. 2001;25:62-74.

11. Benkorah AY, McMullen JN. Biconcave coated, centrally perforated tablets for oral controlled drug delivery. J Control Release 1994. 1994;32:155-60.

12. Kim CT. Compressed donut shape tablet with zero order release kinetic. Pharm Res. 1995;12:1045-8.

13. Kim CT. Controlled release from triple layer, donut-shaped tablets with enteric polymers. AAPS PharmSciTech. 2005;6:E429-36.

14. Lau OL. Effect of growing season, harvest maturity, waxing, low $\mathrm{O}_{2}$ and elevated $\mathrm{CO}_{2}$ on flesh browning disorders in 'braeburn' apples. Postharvest Biol Technol. 1998;18:131-41.

15. Dou H, Ismail MA, Petracek PD. Reduction of post-harvest pitting of citrus by changing wax by changing wax component and their concentration. Proc Fla State Hort Soc. 2000;112:159-63.

16. Mollohan K, Makhlouf JM. Wax-containing powder coatings, US Patent 3,872,040.

17. Dumortier G, Grossiord JL, Agnely F, Chaumeil JC. A review of poloxamer 407 pharmaceutical and pharmacological characteristics. Pharm Res. 2006;23:2709-28.

18. Adegboye TA, Itiola OA. Physical and release properties of metronidazole suppositories. Trop J Pharm Res. 2008;7:887-9.

19. Chetty P. Development and assessment of propranolol sustained release dosage forms separately and in combination with hydrochlorothiazide. South Africa: Rhodes University; 2006.

20. MicroMath Scientist Handbook Rev., Salt Lake City, UT. USA., 7EEF, MicroMath, 1995, p. 467.

21. Choncheewa C, Phaechamud T. Characterization and drug behavior in shellac wax-poloxamer matrix tablets fabricated with mold technique. J Metal Mater Miner. 2012;22:67-74.

22. Zema L, Loreti G, Melocchi A, Gazzanida A. Injection molding and its application to drug delivery. J Control Release. 2012;159:324-31.

23. Phaechamud T. Variable influencing drug release from layered matrix system comprising hydroxypropyl methylcellulose. AAPS PharmSciTech. 2008;9:668-74.

24. Efentakis M, Politis S. Comparative evaluation of various structures in polymer controlled drug delivery systems and the effect of their morphology and characteristics on drug release. Eur Polym J. 2006;42:1183-95.

25. Kolašinac N, Kachrimanis K, Homšek I, Grujić B, Đurić Z, Ibrić S. Solubility enhancement of desloratadine by solid dispersion in poloxamers. Int J Pharm. 2012;436(1-2):161-70.

26. Hu J, Johnston KP, Williams RO. Rapid release tablet formulation of micronized danazol powder produced by spray-freezing into liquid (SFL). J Drug Delivery Sci Technol. 2004;14(4):305-11.

27. Kojarunchitt T, Hook S, Rizwan S, Rades T, Baldursdottir S. Development and characterization of modified poloxamer407 thermoresponsive depot systems containing cubosomes. Int $\mathbf{J}$ Pharm. 2011;408:20-6.
28. Lee B-J, Ryu S-G, Cui J-H. Controlled release of dual drugloaded hydroxypropyl methylcellulose matrix tablet using drugcontaining polymeric coatings. Int J Pharm. 1999;188:71-80.

29. Conte U, Maggi L. Modulation of the dissolution profiles from Geomatrix ${ }^{\circledR}$ multi-layer matrix tablets containing drugs of different solubility. Biomaterials. 1996;17:889-96.

30. Missaghi S, Patel P, Tiwari SB, Farrell TP, Rajabi-Siahboomi AR. Investigation of the influence of tablet shape, geometry and film coating on drug release from hypromellose extended-release matrices. Drug Dev Delivery. 2010;10:32-41.

31. Pandit NK, Wang D. Salt effects on the diffusion and release rate of propranolol from poloxamer 407 gels. Int J Pharm. 1975;167:183-9.

32. Zahirul M, Khan I. Dissolution testing for sustained or controlled release oral dosage forms and correlation with in vivo data: challenges and opportunities. Int J Pharm. 1995;140:131-43.

33. Domańska U, Pobudkowska A, Pelczarska A, Żukowski L. Modelling, solubility and $\mathrm{pK}_{\mathrm{a}}$ of five sparingly soluble drugs. Int J Pharm. 2011;403:115-22.

34. Lee MS, Huang CL, Huang SH, Chen YP, Chen CJ, Wen KC. A comparative study on the dissolution profiles of commercial hydrochlorothiazide tablets. J Food Drug Anal. 2002;10:18-24.

35. Yadav G, Bansal M, Sargam NT, Khare P. Multiple tablets and their drug release kinetic models for oral controlled drug delivery system. Middle-East J Sci Res. 2013;16:782-95.

36. Abdul S, Poddar SS. A flexible technology for modified release of drugs: multi layered tablets. J Control Release 2004. 2004;97:393-405.

37. Ahmed SI, Mangamoori LN, Rao YM. Formulation and characterization of matrix and triple-layer matrix tablets for oral controlled drug delivery. Int J Pharm Pharm Sci 2010. 2010;2:137-43.

38. Qiu Y, Chidambaram N, Flood K. Design and evaluation of layered diffusional matrices for zero-order sustained-release. J Control Release. 1998;51:123-30.

39. Tajarobi F, Larsson A, Matic H, Abrahmsén-Alami S. The influence of crystallization inhibition of HPMC and HPMCAS on model substance dissolution and release in swellable matrix tablets. Eur J Pharm Biopharm. 2011;78:125-33.

40. Vynckier AK, Lin H, Zeitler JA, Willart JF, Bongaers E, Voorspoels $\mathrm{J}$, et al. Calendering as a direct shaping tool for the continuous production of fixed-dose combination products via co-extrusion. Eur J Pharm Biopharm. 2015;96:125-31.

41. Lin Q, Fu Y, Li J, Qu M, Deng L, Gong T, et al. A (polyvinyl caprolactam-polyvinyl acetate-polyethylene glycol graft copolymer)-dispersed sustained-release tablet for imperialine to simultaneously prolong the drug release and improve the oral bioavailability. Eur J Pharm Sci. 2015;79:44-52.

42. Hardy IJ, Windberg-Baarup A, Neri C, Byway PV, Booth SW, Fitzpatrick S. Modulation of drug release kinetics from hydroxypropyl methyl cellulose matrix tablets using polyvinyl pyrrolidone. Int J Pharm. 2007;337:246-53.

43. Machín R, Isasi JR, Vélaz I. Hydrogel matrices containing single and mixed natural cyclodextrins. Mechanisms of drug release. Eur Polym J. 2013;49:3912-20. 